\title{
Minoritized Females in Athletic Training: Educator and Clinician Perspective on Exploring Barriers to Healthcare Delivery
}

Toni M. Torres-McGehee, PhD, SCAT, ATC*; Nancy A. Uriegas, MS, SCAT, ATC*; Kenya Moore, MS, SCAT, ATC $^{\dagger}$

*University of South Carolina, SC, †Columbia College, SC

Key Phrases

Diversity, inclusion, equity, health disparities, patientcentered care

\section{Correspondence}

Dr. Toni M. Torres-McGehee, University of South Carolina, Blatt PE Center-EXSC, Columbia, SC 29208.

E-mail: torresmc@mailbox.sc.edu

Twitter: @torres_mcgehee

\section{Full Citation}

Torres-McGehee TM, Uriegas NA, Moore K. Minoritized females in athletic training: Educator and clinician perspective on exploring barriers to healthcare delivery. Clin Pract Athl Train. 2021;4(1): 1-7.

https://doi.org/10.31622/2021/0004.1.1.

Submitted: January 23, 2021 Accepted: February 11, 2021

\section{EDITORIAL}

In the last 5 years, there have been lessons learned about our country. A lesson of history, in general, is that disunity poses a threat to healthcare; our general health is a product of the connections that we share as individuals and healthcare providers. The COVID-19 pandemic has elevated the importance of these connections. This means we all need to work towards a collective vision of health and peace at all levels. This vision must be built on values of equity, mutual respect, openness, trust, and tolerance. To be successful, we must focus on creating communities that are welcoming to everyone, including those with perspectives that differ from our own. This requires us, as individuals and as a community, to rise above and set a clear example to our faculty, students, patients, and others of how a strong inclusive society operates, both professionally and personally, thus, enhancing our healthcare systems and patient-centered care for athletic training. Specifically, perspectives from minoritized females as healthcare providers and educators are critical in understanding the challenges as well as the positive endeavors that make us successful in our roles.

\section{CULTIVATING CULTURAL PROFICIENCY THROUGH EDUCATION: STORIES FROM A MINORITIZED EDUCATOR}

As a female Mexican-American faculty member at a large public institution, I acknowledge that there are many challenges we face as minoritized female faculty members at a predominantly White institution. However, there are also positive experiences starting with the environment of your institution. A positive environment values diversity, equity, inclusion, and social justice and there is a support system in place for minoritized faculty, staff, and students. The location where your athletic training program is housed may impact the education and research opportunities for faculty and students. From personal experience, being housed in an accredited School of Public Health allows for minoritized faculty and our athletic training programs to prepare professional practitioners and scholars to best serve our communities and impact disease prevention through healthcare, public health education, and intervention. It also provides a platform for faculty and students to collaborate in interprofessional practice, education, and research with public health professionals and healthcare providers. These collaborations improve the lives of individuals, families, communities, and diverse populations through a variety of educational and research endeavors (e.g., health inequalities, children's physical 
activity, community health workers, Latino immigration studies, disability research, global health, speech and hearing, nutrition, exercise, rural and minority health).

It is our mission to work closely with the communities that we serve to provide the best patient-centered care to our underserved populations. Through our work (i.e., teaching, research, and service) and patient care we have an opportunity to advocate for making an impact on changing socioeconomic marginalization, disparities in healthcare, and racial injustice. We do this by educating our students on the diversity, equity, inclusion, and the concepts of cultural competence and cultural humility. These are all critical components of patient-centered care. Diversity in medical education signifies inclusiveness, mutual respect, and multiple perspectives and serves as a mechanism for change resulting in health equity. ${ }^{1}$ In this context, diversity includes all aspects of human differences such as race, ethnicity, religion, national origin, age, marital status, disabilities, sexual orientation, gender, gender identity and expression, and socioeconomic status. Inclusion can be achieved by fostering an understanding of culture and climate of your organization or within patient care settings.

Culture competence highlights the demand for health care system and providers to be aware of and responsive to patient's cultural perspectives and backgrounds; ${ }^{2}$ and requires healthcare providers to appreciate and respect the patient's individual viewpoints. Cultural competency also encourages awareness of health disparities and discrimination, which is a skill that can be taught, trained, and achieved and is often described as essential for working effectively with diverse patients to improve access to healthcare, increase health literacy, improve health care quality, and promote health equity. ${ }^{3-5}$ Cultural humility is often confused with cultural competence; these terms are defined independent of each other but can be used at the same time. For example, cultural humility involves entering a relationship with another person with intentions of honoring their beliefs, customs, and values. ${ }^{2}$ This is an on-going process of self-reflection and self-critique, combined with the willingness to learn from others and addressing power imbalances and avoidance of stereotyping. When we merge these two concepts, this allows for a more meaningful connection with each patient as a unique individual, with diverse perspectives, culture, and lifestyles. This concept was coined cultural competemility, ${ }^{3}$ which is a synergistic process between cultural humility and cultural competence in which cultural humility permeates each of the five components of cultural competence: cultural awareness, cultural knowledge, cultural skill, cultural desire, and cultural encounters. ${ }^{3}$ Faculty, students, and practitioners must be cognizant of these concepts and how they all intersect with patient-centered care, student-centered learning, and research collaborations.

Our healthcare system is professionally driven toward patient-centered care, which incorporates an individual's perspective and more involvement in his/her/their care results in better health outcomes and satisfaction. ${ }^{6}$ However, it is important to note there are certain populations such as low-income individuals, uninsured persons, immigrants, racial and ethnic minorities, veterans, the disabled, and the elderly who are typically underserved by the health system. Thus, in turn, facing greater barriers to patient-centered care. To best tackle these challenges, it is critical to collaborate outside your organization and develop community partners. A goal of our program is to expose our faculty, postprofessional athletic trainers, and professional athletic training students to a variety of patient populations and settings (i.e., Historically Black Colleges and Universities, private colleges, public colleges/universities, private and public high schools, rural private and public high schools, and inner-city schools). 
To be successful in these environments, it is critical to prepare young professionals prior to their clinical placements and provide on-going mentoring while they are providing patient care. Often young professionals are not aware of the social determinants of health in the population that they work with. Therefore, preparation begins with onboarding activities to include but are not limited to reviewing issues of diversity, equity, inclusion, social justice, how to become culturally proficient; ways to combat racism, and identify social determinants of health, healthcare, and health disparities within the community the serve. It is recommended, administrators integrate an activity during onboarding related to the clinician/student conducting thorough assessment on the community/city they serve (Table 1). With most of our students being from out-of-state, this not only allows them to familiarize themselves with the community/city, but also allows them to identify social determinants of health, potential resources, community collaborations and identify other healthcare practitioners to engage in interprofessional practice. This may also be conducted in a field trip manner. ${ }^{7}$ Developing relationships with community partners allows for the integration of best practices with patient care and opens opportunities for research between clinicians, faculty, and marginalized communities. Building trust and mutual respect with these communities, allows for ethical recruitment of marginalized or vulnerable groups. In turn, this allows successful research in the identification of patient health care needs for each population and organization.

- Toni M. Torres-McGehee, PhD, SCAT, ATC

\section{PATIENT CARE PERSPECTIVES IN THE CLINICAL SETTING}

As a black woman in health care, I have been on the receiving end of the negative preconceived notions, discrimination, and microaggressions that

\section{Table 1: Exploring the Community you Serve}

1. Identify community/city population and distribution of race/ethnicity

2. Identify community/city cost of living

3. Identify community/city average household income

4. Familiarize yourself with surrounding neighborhoods, parks, and shopping areas

5. Familiarize yourself with public transportation

6. Identify types of community/city health services (e.g., financial support, communication resources such as speech and language services, behavioral resources, sensory and motor services, social and recreational services, family support services, school services, research opportunities, etc.)

7. Identify health care facilities (e.g., hospitals, clinics, urgent care, veteran services, dentists, optometrist, physical therapy, occupational therapy, disability services, specialty clinics, social work, counselors, etc.)

8. Identify current community/city social determinants of health data (e.g., social and community, neighborhoods/environment, demographics, educational attainment, economics, insurance coverage, health status - disabilities, heart disease, chronic disease, etc.)

9. Identify the location of public library

10. Identify communities of faith (religious facilities)

11. Identify locations of private and public schools

12. Identify cultural events in the community/city

are projected onto minoritized populations. As humans we all wrongly assume things about those we do not know or understand. However, for too long the distorted images of people of color and minoritized persons that stem from racism and stereotypes, have been supported not only by individuals but also by institutions like our government, educational system, healthcare, and workplace. Within these institutions, we find that 
policies and laws, were by design, created to exclude. Systemic oppression creates barriers that are often covert, and inherently carry negative messages to minoritized persons about our "place" in society and what is or is not accessible to us. I recall stories from my parents about their experiences as children. Enduring everything from racial slurs to the struggles of segregation and integration. Because I was surrounded by a loving community and such a rich culture, my eyes were often blind to some of those racial tensions. But as a high school student my heart filled with many emotions as I marched for the first time to protest the murder of Trayvon Martin, a murder that that took place near my home in a neighborhood I had been several times to visit with family friends.

Fast forwarding to 2020, and our country's issues with police brutality towards people of color are still high. It is a very sad reality that some believe lives like mine are expendable or do not matter enough. These physical, emotional, and psychological traumas add to the subliminal messages that ring through society about race. I have experienced times of being racially profiled in stores, and as a college student even been asked where I learned to speak and if there were others like me where I come from. Being categorized as "exceptional for a Black student," was concerning as to suggest students of color are not bright, articulate students. The stigma of having to "work twice as hard" just to be on a level playing field seemed all too true. Usually being one of few students of color in my academic spaces illuminated the impression that I had to carry my culture alone. It highlighted this misconception, that what I do or how I perform will positively or negatively affect the way that others like myself would be perceived and accepted in those spaces. And while one's presence may aid in opening the door for those to come, minoritized persons certainly have the weight of feeling the pressure to succeed. For success was not only for one's self, but for others as well.
As a clinician today, I recognize how those feelings and encounters I faced have led me to be more mindful in the way I practice and interact with my patients. I began working at a low socioeconomic school in South Carolina, where most of the student-athletes were Black. Although I felt I could relate to the students I did not want to assume that I knew them. I did not want to take for granted that I knew their story, what their home life was like, nor what their passions were, or what they valued in life. I was intentional about being visible in the school, getting to know not only the athletes and coaches, but also people like the custodians, nurses, bookkeeper, receptionist, parents, and campus police. Establishing those relationships allowed me to better understand the overall culture, and advocate for the student athlete's health. I often verbalized my treatment plans and involved both the student, parent/guardian, team physician and coaches into the plan of care. I explained why I did something and made room for them to ask questions regarding their own health and progress. I consistently led with openness and transparency, as I wanted each student to feel like they were involved in what was happening to them. If I could, I would accompany students when seeing our collaborating physician to help them feel at ease because for many of them doctors represented people who were just there to capitalize on their pain and provide services they could not afford. I tried to take my time to dismantle the social disparities in health care experienced by my population. And while it was not my role to solve every issue in my time there, my efforts helped set the standard for what those student athletes now expected from health care professionals and improved the care that was available to them.

Regardless of the setting, becoming more aware of the patient population aids in establishing appropriate relationships with those we serve. This ultimately helps guide the process of decisionmaking that considers and respects their beliefs, 
values, language, and traditions. It has caused me to even evaluate what I tolerate as acceptable behavior and speech in the athletic training facilities. As one who is both ethnically diverse and a healthcare provider in athletic training, I recognize the influence I have in igniting change where I am. I have experienced firsthand the benefits and power of representation, mentorship, and leadership from minoritized athletic trainers as well as the benefits of diversity regarding collaboration and innovation. When we think about research and how it leads the way in creating the standards of how we care for our patients, overlooking the importance of diversity, even here, can be detrimental. Most research in the realm of athletic training does not currently reflect such inclusion, having shown to provide gaps in applicability. As clinicians we talk about evidence-based practice and integrating available research, merging that with clinical knowledge. This should include efforts in both addressing a lack of diversity amongst research participants involved, as well as research that gains the perspectives of diverse certified and athletic training students.

It may be easier said than done to operate in a way where we are proficient in interacting with those across an array of backgrounds. So, how do we transition from just merely having a "one size fits all" mentality in our care to one that is a lot more progressive and considerate of our patient's needs, background, health concerns, health beliefs, and values? Historically, there have been many systemic barriers that exist for minoritized persons pertaining to health care, along with other racial disparities that perpetuate discrimination and stereotypes. We must learn to come from a place of empathy and compassion with a desire to understand our patients. Additionally, there must be acknowledgment of any biases that hinder us from effectively treating others or that cause us to treat one group different from another. These steps are key to change from the inside out. It will take a willingness to be uncomfortable, as there is always difficulty with having to realize biases thoughts and feelings that sit within the subconscious mind.

- Kenya Moore MS, SCAT, ATC

\section{BLENDING CULTURES WITHIN PATIENT- CARE SETTINGS}

Through my academic and professional career, I have been an athletic training student, an undergraduate teaching assistant, a secondary school athletic trainer, a performing arts athletic trainer, a military athletic trainer, a doctoral research and teaching assistant and in each setting - a minoritized person. Twenty years ago, if you were to ask my parents what they expected of their children regarding education, they would both state, and I translate: "to accomplish more than I did." I am the daughter of two Mexican born and raced individuals, one whom completed 4 th grade and the other not much further, 6th grade. The resources were so limited that they had one classroom and one teacher to teach students regardless of their grade level or age; and where 6th grade was the highest level of education you could attain. Living there, never did high school cross their minds, and let us not even mention college, because odds are, they had no idea what that was or how to even get there. If asked that same question ten years ago and knowing what a college education meant, that was the new expectation; an expectation soon to be met by all five children of a meagerly educated Mexican couple. In 2015, I became a 1 st generation college graduate; that same year my oldest sister and brother received their master's degree. It did not stop there; today I am a second year PhD student and scholar in the making.

Following my education and clinical rotations at an institution with many resources available at hand, I began my journey as a clinician in the same 
community I came from a predominately Spanish speaking and low-income city on the Mexican border. Health literacy is extremely low and often I found myself translating orthopedic or concussion associated terminology to patients and parents, which is quite difficult for anyone who went through an English-only curriculum, even when Spanish is your first language, and you speak it daily. Information always had to be simplified, and though difficult, I was comfortable; I was around those that looked like me, talked like me, and shared the same culture and values as me. The students could reflect on me and I could reflect on them. I was once in their shoes and the concerned parents reminded me of my own mother driving me to urgent care to get an X-ray because that is what you do when your ankle looks like a balloon, right? It was here I learned the importance of patient education, whether that was in English or in Spanish. I learned to have open discussions about their injuries, their goals, their accessibility to care and medical insurance, and what the best plan of care and treatment would be for them. It was here I learned to be patient centered, around the same community I grew up in, around the same struggles $I$, as a patient, faced.

I transitioned to practice across the country in a small rural high school in St. Matthews, South Carolina, where the state's Hispanic or Latino population is less than $6 \%$. I was an outsider; my patients constantly asked if I played soccer growing up, because "that is the only sport the Hispanic kids play here." As much of an outsider as I was, there were some things that remained the same-my work was in the best interest of my patients and their well-being. I was now in a predominately Black school, coaches were predominately White males, and the community was close knit; I was no longer in my comfort zone. Head coaches constantly questioned and undermined me, parents always asked where I was from; people around me said I spoke "funny." One of my biggest challenges was being told I was not a doctor and my medical opinion did not count compared to that of a nurse practitioner who signed a clearance note. Once again, education was my most powerful tool. I do not think coaches ever expected a female athletic trainer to hold her ground, as I did; and to make it known that my number one priority was the health of the patients.

All the patients had different upbringings from mine, but in some ways so similar. I submerged myself into the community there too, I made it known that my job was not $2-7 \mathrm{pm}$ and that where I came from did not mean I did not understand what they were going through. The greatest appreciation came from parents; I gained their trust simply through education and patientcentered care. We learned about each other in the process; we respected our diverse backgrounds and adapted to what their needs were. When the resources were not available, I went out of my way collaborating with our collaborating physician and orthopedic network to provide as much as we could for them. What goes unnoticed in these settings is the power of research, constant self-assessment, and patientreported outcome measures. To bring change and more resources, others must see the value of our work. Lastly, a few things we always kept in mind: an open-door always meant you were welcomed in, respect was reciprocated and practiced daily, and patient care and goals were the priority. I learned that the patient-clinician rapport can only evolve with continuous communication, trust is earned, and happiness in the workplace is not dependent on the resources available but network you build in the community.

- Nancy A. Uriegas MS, SCAT, ATC

\section{SUMMARY}

Our experiences are diverse and unique in their own way. However, all three of us have faced similar challenges as minoritized female clinicians and educators. We have also seen the challenges 
faced by our diverse patients and students. We as an athletic training profession, should promote and practice diversity, equity, inclusion, and social justice across our settings. We must come together, rise above, and lead by example and this begins with providing opportunities of education, research, and patient-centered care equally across our communities. Let us all take a step back, evaluate where we are, who we serve, and how we can improve in the best interest of those around us.

\section{REFERENCES}

1. Nivet MA, Castillo-Page L, Schoolcraft Conrad S. A diversity and inclusion framework for medical education. Acad Med. $2016 ; 91(7): 1031$.

2. Betancourt JR, Green AR, Carrilo JE. Cultural competence in health care: Emerging frameworks and practical approaches. 2002. https://www.commonwealthfund.org/sites/d efault/files/documents/media files publ ications fund report 2002 oct cultural com petence in health care emerging framew orks and practical approaches betancourt culturalcompetence 576 _pdf.pdf.

3. Campinha-Bacote J. Cultural competemility: A paradigm shift in the cultural competence versus cultural humility debate, Part I. Online J Issues Nurs. 2019;24(1).

4. Powell DL. Social determinants of health: Cultural competence is not enough. Creat Nurs. $2018 ; 24(1): 5-10$.

5. Wong WF, LaVeist TA, Sharfstein JM. Achieving health equity by design. Jama. 2015;313(14):1417-1418.

6. Rathert C, Wyrwich MD, Boren SA. Patientcentered care and outcomes: a systematic review of the literature. Med Care Res Rev. $2013 ; 70(4): 351-379$.

7. Chang AY, Bass TL, Duwell $M$, et al. The impact of "see the city you serve" field trip:
An educational tool for teaching social determinants of health. J Grad Med Educ 2017;9(1):118-122. 